\title{
The interference effect of concurrent working memory task on visual inhibitory control
}

\author{
Brad M. Hong \\ The Pingry School \\ Basking Ridge, NJ, USA \\ Michael Clancy
Drew University
}

\author{
Susan Weiner \\ Drew University \\ Madison, NJ, USA \\ Minjoon Kouh* \\ Drew University
}

\begin{abstract}
We examined the interference between inhibitory control of a saccadic eye movement and a working memory task. This study was motivated by the observation that people are susceptible to cognitive errors when they are preoccupied. Subjects were instructed to make an anti-saccade, or to look in the opposite direction of a visual stimulus, thereby exercising inhibitory control over the reflexive eye movement towards a salient object. At the same time, the subjects were instructed to memorize a random sequence of digits that were read out to them, thereby engaging their working memory. We measured the success of an eye movement by rapidly switching between images and asking the subjects what they saw. We found that these concurrent cognitive tasks significantly degraded antisaccade performance.
\end{abstract}

Keywords: eye movement, eye tracking, saccades, antisaccades, working memory, inhibitory control, prefrontal cortex

\section{Introduction}

With today's media-rich lifestyle, people are often dividing their attention and cognitive capacity into multiple tasks. For example, many students do their homework while chatting online, or people manipulate their smartphones while driving. While performing those tasks, the prefrontal cortex (PFC) is engaged in executing cognitive control, in storing task-relevant information in working memory, and in exercising inhibitory control as needed (Baddeley, 1992; Miller \& Cohen, 2001; Roberts et al., 1994). How such different cognitive functions are coordinated and executed is an open question in neuroscience. Related to this question is how different neural

Received February 4, 2016; Published April 27, 2016.

Citation: Hong, B. M., Weiner, S., Clancy, M., \& Kouh, M. (2016).

The interference effect of concurrent working memory task on visual inhibitory control. Journal of Eye Movement Research, 9(3):5, 1-5.

Digital Object Identifier: 10.16910/jemr.9.3.5

ISSN: $1995-8692$

This article is licensed under a Creative Commons Attribution 4.0 International license. (cc) B EY operations interact or even interfere with each other, increasing susceptibility to cognitive errors during multitasking.

We closely followed the experimental protocol by Roberts et al. (1994), and explored the interference effect between two cognitive tasks. One is an anti-saccade task (Hallett, 1978; Roberts et al., 1994), and the other is to remember a sequence of random digits for a few seconds.

A saccade is a rapid eye movement from one point of fixation to another, which brings the fovea-a small yet high-resolution part of the retina-into different regions of the visual field at roughly three times per second. Saccades can be triggered reflexively, in response to a salient visual feature (e.g., colors or textures that are distinct from the background) or a conspicuous movement in a visual scene. When a person is presented with a distinctive cue in the visual periphery, a natural, reflexive response is to make a "pro-saccade" by moving her gaze towards it. However, she can exercise inhibitory control over the saccadic reflex and to make an "anti-saccade," or look towards the opposite side of the stimuli. 
Because of the finite capacity of the neural hardware, overloading working memory leads to decreased inhibitory control. An analogy would be the sluggish performance of an old computer that is running a memoryintensive program. Patients with prefrontal lesions or dysfunctions, such as schizophrenia (Fukushima et al., 1990), Alzheimer's disease (Fletcher \& Sharpe, 1986), and attention deficit hyperactivity disorder (Aman et al., 1998), tend to commit more errors in tasks that require inhibitory control or working memory.

Studies suggest that the superior colliculus (SC) of the brain is involved in the reflexive saccade (Schiller \& Sandell, 1983) and it receives projections directly and indirectly from the dorsolateral PFC (Goldman-Rakic, 1988). A number of event-related fMRI studies have shown the involvement of PFC during an anti-saccade task (Ettinger et al., 2008; Ford et al., 2005). We speculate that during an anti-saccade task, the PFC provides an inhibitory signal to the SC to prevent an unwanted reflexive saccade. However, if the PFC is occupied with another task, the inhibitory signal may not be sent, and the reflexive saccade would be more likely than an antisaccade. Hence, it is our hypothesis that by overloading the capacity of the PFC with two tasks requiring inhibitory control and working memory, average performance would decrease.

\section{Methods}

College-aged subjects $(\mathrm{N}=11)$ participated in our study. The experiments were conducted under an Institutional Review Board (IRB) approved protocol. Each subject performed three types of tasks: pro-saccade with the working memory task (PM), and anti-saccade tasks with and without the working memory task (AM and A).

For each trial, subjects started by looking at a computer monitor with a central fixation point. A sequence of 8 random digits (e.g., “32021287”) was read out by a computer. (In a pilot study, it was observed that a short sequence like a 3-digit number did not require much effort to memorize perfectly, and did not produce any significant interference effect.) After a variable interval (between 1500 and $3500 \mathrm{~ms}$ ), the fixation point was eliminated and a white square (cue) appeared on either the right or left side for $200 \mathrm{~ms}$, during which the subjects were expected to make a saccadic eye movement towards or away from the cue, respectively for pro-saccade or anti-saccade tasks. Then, the cue disappeared, and a small arrow (target), pointing either up or down, was shown for $110 \mathrm{~ms}$ on the appropriate side of the monitor. The target was presented at 15 degrees of visual angle from the center of the screen. The size of the cue and masking squares was $1 \times 1$ degrees. The size of the arrow was 0.5 degree, fitting snugly inside the square. Given this eccentricity and size, the arrow was not clearly visible when subject's gaze was on the fixation point.

The target appeared on the same location as the cue for the PM trials and on the opposite side of the cue for AM or A. Because the target arrow appeared only briefly and because it was too small to be seen clearly with peripheral vision, an incorrect eye movement (i.e., a prosaccade during an anti-saccade trial) would not allow the subjects to identify the direction of the arrow. The target was then replaced by a gray square (mask) for another $200 \mathrm{~ms}$, so that subjects would not be able to recognize the direction of the arrow by the persistence of vision. Figure 1 shows the experimental design.

Subjects were instructed that the eye movement should be made as soon as the cue appeared. If a correct eye movement were made, the subjects would have been able to see the direction of the arrow, which appeared in a brief time interval between the cue and the mask. Following the visual task, subjects had to choose one of the following three choices with a keyboard about the direction of the arrow: Up, Down, or Unsure. The correctness of this keyboard response was used to measure the saccade performance. Subjects also had to type in the sequence of random digits, as best as their memory served. In trials without the memory task (A), the number sequences were still played, but subjects were instructed to ignore the audio, and they reported the direction of the arrow only.

Each subject performed 144 trials in 9 sets of 16, lasting a total of approximately one hour. Cue location (left or right) and arrow direction (up or down) were counterbalanced. Each subject performed three sets of each trial type in the following progression: $\mathrm{PM}>\mathrm{A}>\mathrm{AM}$. Easier pro-saccades trials preceded less familiar anti-saccade trials, so that subjects could become acclimated to the experiments.

The stimuli were presented on a 17-inch computer monitor with a resolution of 1280 by 1024 pixels at 60 Hz. Subjects' eyes were about $60 \mathrm{~cm}$ away (at about an 
arm's length) from the monitor. Matlab R2012b was used to present both visual and auditory stimuli and to collect keyboard responses.

We note that in performing this experiment, there is no explicit need for an eye-tracker. By switching visual stimuli quickly enough and by ensuring that the target (i.e., arrows) cannot be seen with a peripheral vision, the success of subjects' eye movements can be measured with a regular computer and a monitor.
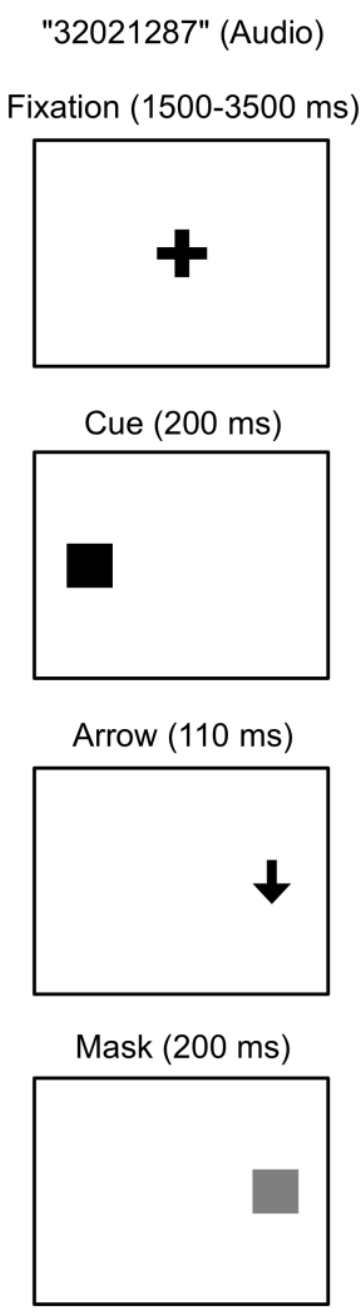

Report (Arrow and Digits)

Figure 1. Sequence of sample visual and auditory stimuli for an anti-saccade trial. The location of the cue (left or right) and the direction of the arrow (up or down) were chosen randomly. For readability, the figures are not drawn to scale.

\section{Results}

The average performance for pro-saccades was $94 \%$ (PM), while the average for anti-saccade was $43 \%$ (A) and $31 \%(\mathrm{AM})$, as shown in Figure 2. The difference between $\mathrm{A}$ and $\mathrm{AM}$ conditions was significant (paired $\mathrm{t}$ test, $p<0.01)$. This is an expected trend, given the cognitive demand of keeping 8 digits in the working memory while inhibiting, rather than following, saccadic reflexes.
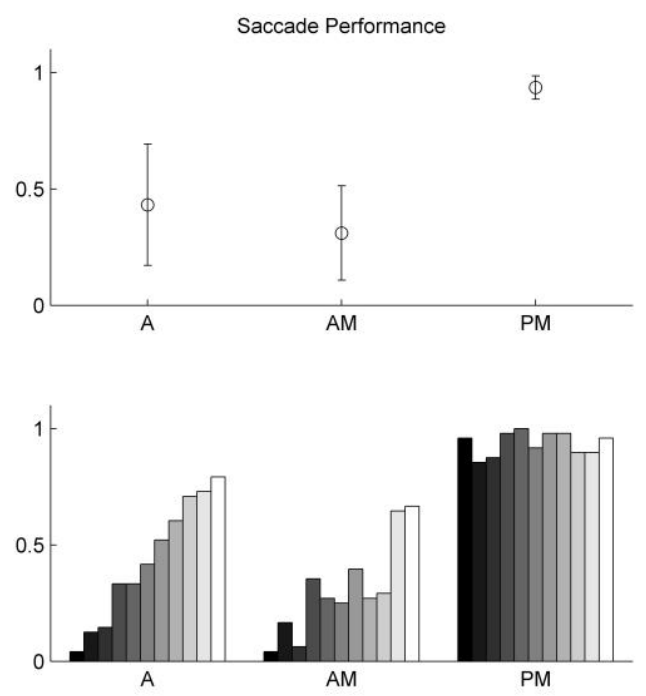

Figure 2. The anti-saccade task (A and $A M)$ is more difficult than the pro-saccade task (PM). The anti-saccade with a concurrent working memory task (AM) is also significantly more difficult than the anti-saccade task alone (A). The average across subjects is shown at the top, and individual subject data is shown at the bottom. Individual subject is coded by the same gray-scale.

On the other hand, the average performances on the memory task were $57 \%(\mathrm{AM})$ and $58 \%(\mathrm{PM})$, as shown in Figure 3, and they were not significantly different (paired t-test, $p>0.1$ ).

There was a high variability across subjects. For example, some subjects' average performances on antisaccade task were over $70 \%$, whereas other subjects performed less than $10 \%$. The memory performance was highly variable, too. Furthermore, these variabilities correlated with each other, as shown in Figure 4. Unsworth et al. (2004) also reported that subjects with higher work- 
ing memory capacity performed better in anti-saccade task.
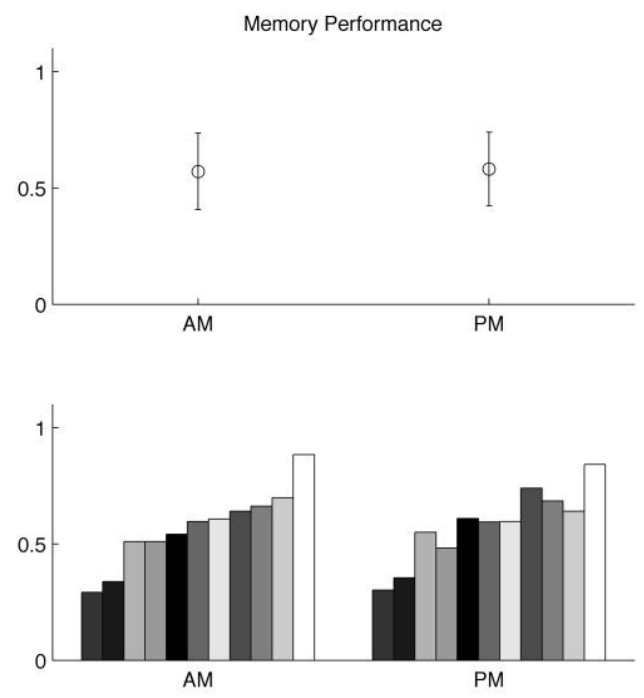

Figure 3. Whether the subjects were performing antisaccade (AM) or pro-saccade (PM), there was no significant difference in the working memory performance. Subject-wide data is shown at the bottom. The performance of 0.5 indicates that the subject was able to recall 4 out of 8 digits correctly. The same gray scale as in Figure 2 codes individual subject.

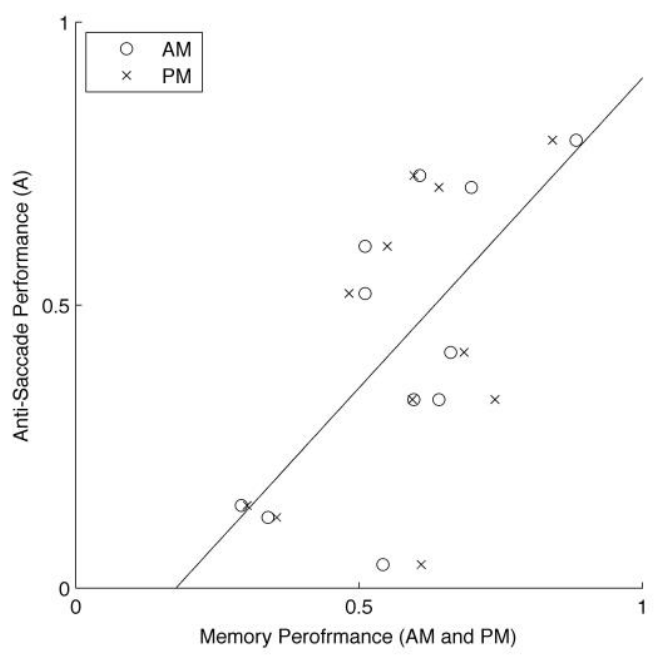

Figure 4. The subjects, who are performing well in the antisaccade task, tend to do well in the working memory task, too. The correlation coefficient between $A$ and AM performances was 0.69 , and 0.54 , between $A$ and PM. The best-fit line between $A$ and $A M$ is shown.

\section{Discussion}

We presented a simple experiment that can measure the success of an eye movement without explicitly measuring the gaze location or requiring an eye tracker. In particular, this study explored how two concurrent tasks, involving inhibitory control and working memory in vision and audition, interacted with each other.

Our subjects' performance was lower than usually reported (e.g., 10\% error rate, as reported by Unsworth et al.), and this is likely due to a limitation of our approach of allowing only a narrow time-window (between 200 and $310 \mathrm{~ms}$ from the cue onset) for correctly looking at the target. If a subject had a slow reaction time, the mask would have replaced the target, even if the subject made the correct eye movement. Therefore, the observed performance based on the target identification is expected to be lower than what it would have been if the actual eye movements were measured, and the actual interference effect is likely to be more than the modest amount shown in Figure 2.

We found an asymmetric interference effect between the two tasks. While the anti-saccade performance decreased significantly, the working memory performance was not affected. This asymmetry could be due to the fact that the anti-saccade is such an unnatural task that it is more sensitive to the capacity of the PFC. Also, while the working memory task might not require inhibitory control, the working memory could be a requisite of the antisaccade task for which the subjects need to keep reminding themselves to look in the opposite direction of a stimulus. It will be interesting to see whether a different pair of tasks could produce an opposite trend (i.e., degraded performance on the working memory task without affecting the inhibitory control). Also, Kirchner and Colonius (2005) found that the anti-saccade performance could be facilitated, or impeded, by presenting an auditory stimulus either at the same or at the opposition location of a visual stimulus.

Another interesting result is the correlation between the anti-saccade and memory performances across the subjects (Figure 4), which is consistent with the idea the degree of engagement or capacity of PFC's executive control is important for the anti-saccade and working memory tasks (Baddeley, 1992; Miller \& Cohen, 2001; Roberts et al., 1994). At the same time, it has been reported that other factors, such as foreknowledge or task- 
switching (Barton et al., 2006; Unsworth et al., 2004), could influence anti-saccade performance.

\section{Contributions}

$\mathrm{BH}$ and $\mathrm{MK}$ designed the experiment. $\mathrm{BH}$ collected pilot data. MK created stimuli, wrote codes for data collection and analysis, analyzed data, and wrote the paper. SW recruited subjects and ran the experiment. MC developed codes for reading out a random sequence of digits.

\section{Acknowledgements}

The authors declare that there is no conflict of interest regarding the publication of this paper.

\section{References}

Aman, C.J., Roberts, R.J. Jr, \& Pennington, B.F. (1998). A neuropsychological examination of the underlying deficit in attention deficit hyperactivity disorder: frontal lobe versus right parietal lobe theories. Developmental Psychology 34(5): 956-969.

Baddeley, A. (1992). Working memory. Science 255(5044): 556-559.

Barton, J.J.S., Kuzin, A., Polli, F., \& Manoach, D.S. (2006) The use of working memory for task prediction: What benefits accrue from different types of foreknowledge? Neuroscience 193: 385-392.

Ettinger, U. et al. (2008). Decomposing the neural correlates of antisaccade eye movements using eventrelated fMRI. Cerebral Cortex 18:1148-1159.

Fletcher, W.A., \& Sharpe J.A. (1996). Saccadic eye movement dysfunction in Alzheimer's disease. Annals of Neurology 38: 364-370.

Ford, K.A., Goltz, H.C., Brown, M.R.G., \& Everling, S. (2005). Neural processes associated with antisaccade task performance investigated with event-related fMRI. Journal of Neurophysiology 94: 429-440.

Fukushima, J., Fukushima, K., Chiba, T., Tanaka, S., Yamashita, I., \& Kato, M. (1988). Disturbances of voluntary control of saccadic eye movements in schizophrenic patients. Biological Psychiatry 23: 670677.
Gazzaniga, M.S., Ivry, R.B., \& Mangun, G.R. (Eds.). (2009). Cognitive neuroscience: The biology of the mind (2nd ed.). New York: W.W. Norton.

Goldman-Rakic, P.S. (1988). Circuitry of primate prefrontal cortex and regulation of behavior by representational memory. In F. Plum (Ed.), Handbook of physiology: The nervous system (Vol. V, pp. 373-417). Bethesda, MD: American Physiological Society.

Hallett, P.E. (1978). Primary and secondary saccades to goals defined by instructions. Vision Research 18 : 1279-1296.

Kirchner, H. \& Colonius, H. (2005). Cognitive control can modulate intersensory facilitation: speeding up visual saccades with an auditory distractor. Experimental Brain Research 166: 440-444.

Lafer-Sousa, R. \& Conway, B.R. (2009). Vision and Art: An interdisciplinary approach to neuroscience education. The Journal of Undergraduate Neuroscience Education 8(1): A10-A17.

Miller, E.K. \& Cohen, J.D. (2001). An integrative theory of prefrontal cortex function. Annual Reviews Neuroscience 24: 167-202.

Miller, G.A. (1956). The magical number seven plus or minus two: some limits on our capacity for processing information. Psychological Review 63(2): 81-97.

Roberts, R.J., Jr, Hager, L.D., \& Heron, C. (1994). Prefrontal Cognitive Processes: Working memory and inhibition in the antisaccade task. Journal of Experimental Psychology: General 123(4): 374-393.

Schiller, P.H. \& Sandell, J.H. (1986). Interactions between visually and electrically elicited saccades before and after superior colliculus and frontal eye field ablations in the rhesus monkey. Experimental Brain Research 49: 381-392.

Unsworth, N., Schrock, J.C. \& Engle, R.W. (2004) Working memory capacity and the antisaccade Task: Individual differences in voluntary saccade control. Journal of Experimental Psychology: Learning, Memory, and Cognition 30(6): 1302-1321. 